\title{
LA-UR- $94-3080$
}

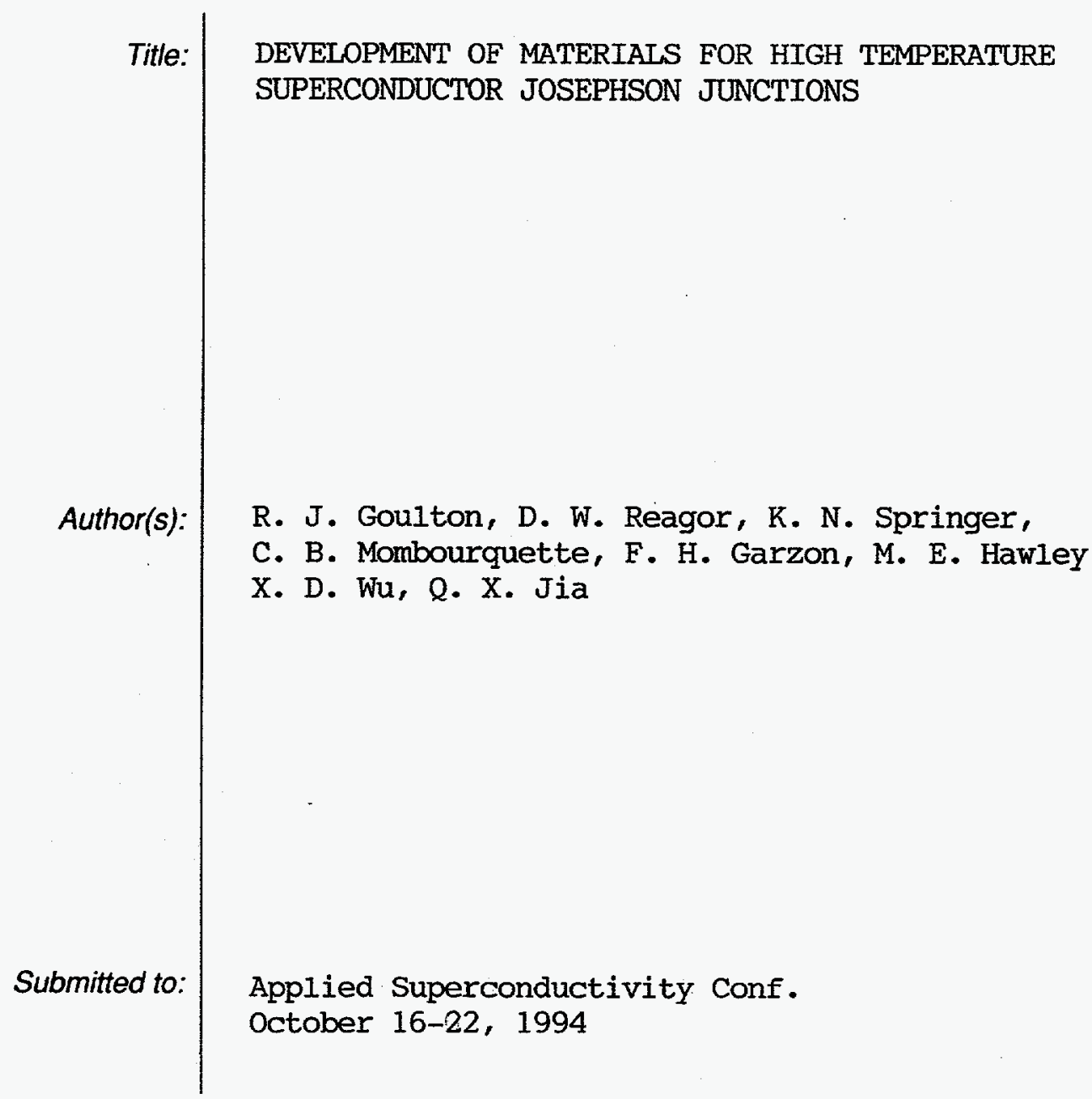

\section{DISCLAIMER}

This report was prepared as an account of work sponsored by an agency of the United States Government. Neither the United States Government nor any agency thereof, nor any of their employees, makes any warranty, express or implied, or assumes any legal liability or responsibility for the accuracy, completeness, or usefulness of any information, apparatus, product, or process disclosed, or represents that its use would not infringe privately owned rights. Reference herein to any specific commercial product, process, or service by trade name, trademark, manufacturer, or otherwise does not necessarily constitute or imply its endorsement, recommendation, or favoring by the United States Government or any agency thereof. The views and opinions of authors expressed herein do not necessarily state or reflect those of the United States Government or any agency thereof.
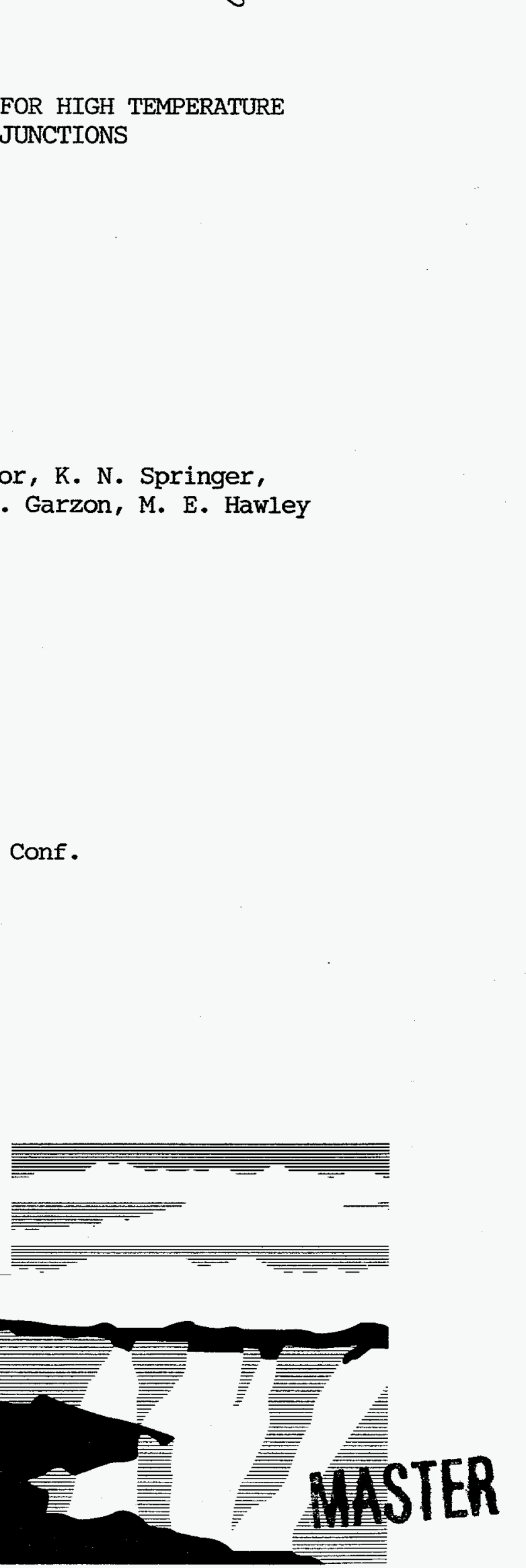

Los Alamos National Laboratory, an affirmative action/equal opportunity emplóyer, is operated by the University of California for the U.S. Department of Energy under contract W-7405-ENG-36. By acceptance of this article, the publisher recognizes that the U.S. Government retains a nonexclusive, royalty-free license to publish or reproduce the published form of this contribution, or to allow others to do so, for U.S. Government purposes. The Los Alamos National Laboratory requests that the publisher identify this article as work performed under the auspices of the U.S. Department of Energy. 


\section{DISCLAIMER}

Portions of this document may be illegible in electronic image products. Images are produced from the best available original document. 


\title{
Development of Materials for High Temperature Superconductor Josephson Junctions
}

\author{
R.J. Houlton, D.W. Reagor, M.E. Hawley, K.N. Springer, Q.X. Jia, C.B. Mombourquette, F.H. Garzon, and X.D. Wu \\ Superconductivity Technology Center, Los Alamos National Laboratory, Los Alamos - New Mexico
}

\begin{abstract}
We have conducted a systematic optimization of deposition parameters for fabrication of multilayered oxide films to be used in the development of high temperature superconducting SNS junctions. These films were deposited by off-axis sputtering using a custom fabricated multi-gun planar magnetron system. Each material and the various combinations of materials were optimized for: epitaxial lattice match, crystal quality, film uniformity, electrical properties, and surface microstructure. In addition to the standard procedures commonly used to sputter deposit epitaxial oxide films, a variety of insitu and exsitu procedures were used to produce high quality multilayer devices, including: varying the nucleation temperature from the actual film growth temperature, location of the substrate during the deposition process, constant rotation of the substrate, and timing of the oxygen anneal. The unprocessed films and devices in process were characterized with Atomic Force Microscopy and Scanning Tunneling Microscopy as well as other common materials characterization techniques. Completed multilayer devices were patterned and packaged for electrical characterization. The relationship between material properties and electrical characteristics will be discussed.
\end{abstract}

\section{INTRODUCTION}

The lithographic processing we employ to produce multilayer devices requires that $\mathrm{YBa}_{2} \mathrm{Cu}_{3} \mathrm{O}_{7}(\mathrm{YBCO})$ films be grown on etched or ion milled $\mathrm{MgO}$ surfaces. Typically, these altered substrate surfaces typically produce poor quality YBCO films with a high percentage of 45 degree misoriented material. In this study we have determined parameters that allow high quality films to be grown on these processed substrates.

Manuscript completed September 1, 1994. This work was supported by the United States Department of Energy.

\section{EXPERIMENTAL}

\section{A. SUBSTRATE SELECTION AND PREPARATION}

Epi-polished $<100\rangle$ cut magnesium oxide substrates (Enprotech) were used throughout this investigation. We have found that any substrate cleaning, recycling, or extended exposure to atmosphere, prior to the deposition, resulted in substandard quality $\mathrm{YBa}_{2} \mathrm{Cu}_{3} \mathrm{O}_{7}$ YBCO films. These findings are similar to those reported by Moeckly et al. [1]. A degradation of the transition onset temperature as well as an increase in the width of the full transition to the superconducting state was seen when YBCO films were deposited on substrates that were exposed to water in the vapor or liquid phase, or on recycled substrates. X-ray phi scans and Scanning Tunneling Microscopy (STM) images of the YBCO films displayed misaligned grains and the rocking curve peaks were broadened also indicating poor film quality for YBCO deposited on these substrates. STM images also show some microstructural disorder and in some instances show surface particulates of unidentified material.

\section{B. DEPOSITION SYSTEM}

We have engineered an off axis sputter deposition system for insitu growth of oxide materials. The system uses multiple guns radially oriented about the vertical axis of the vacuum chamber with the sample heater mounted on a pedestal in the center of the enclosure. The perpendicular target-sample geometry is required for the growth of high quality superconductor films (Fig.1). The presence of multiple guns in the chamber enables sequential depositions without exposing the samples to the ambient environment. Both RF and DC magnetron sputtering techniques are performed in this system.

The heater assembly consists of a removable tungsten filament quartz bulb heat source and a nickel sample mounting baseplate. The heating fixture is connected to a rotating shaft that can be oscillated up to 


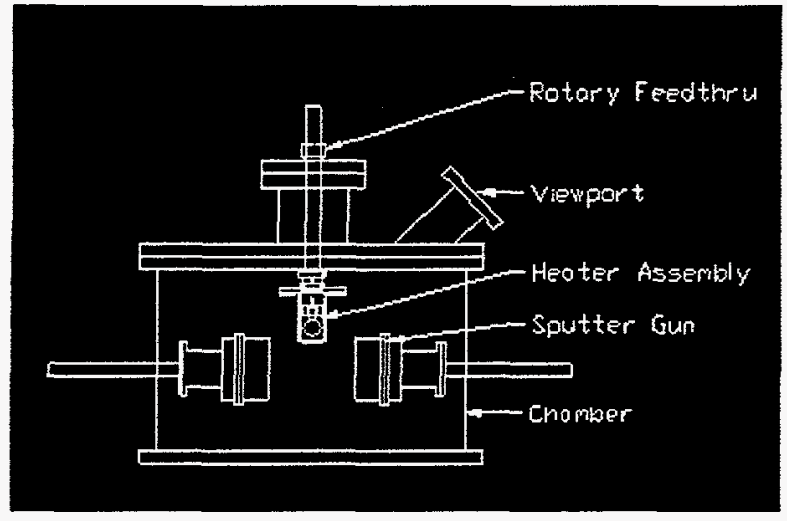

Fig. 1 - Multi-gun sputtered film deposition system .

360 degrees. By oscillating rather than continuously rotating we allow for electrical connections to be hardwired directly to the heater itself, without use of a continuous rotary feedthrough. The oscillations can be continuous or at timed intervals periodically throughout the deposition process. Temperature control is achieved by a digital P.I.D. controller that utilizes a thermocouple embedded in the nickel faceplate of the heater. The substrates are affixed to the faceplate with silver paste.

\section{YBCO GROWTH PARAMETERS AND CHARACTERIZATION}

In a previous publication we studied $\mathrm{YBCO}$ growth on virgin $\mathrm{MgO}$ substrates [2]. In this study, we examine the growth of oxide films on processed $\mathrm{MgO}$ substrates for use in fabricating Superconductor-normalsuperocnductor (SNS) Josephson junction devices. Scanning Tunnelling Microscopy, Atomic Force Microscopy (AFM), Rutherford Backscattering Studies (RBS), and X-ray diffraction $\theta-2 \theta$, rocking curves, and texture analysis techniques were used to evaluate the quality of the deposited materials.

C-axis oriented YBCO films were obtained by using a sputter gas consisting of 160 milliTorr of argon and 40 millitorr of oxygen and a deposition temperature range that varied from $645^{\circ} \mathrm{C}$ to $725^{\circ} \mathrm{C}$. Due to the instability of the YBCO phase in the presence of high partial pressures of oxygen at temperatures above $600^{\circ} \mathrm{C}$ [3], the chamber is held at the deposition pressure while the sample is allowed to cool below $500^{\circ} \mathrm{C}$; then the belljar is flooded to a pressure of 250 Torr of oxygen. The temperature is then maintained at $450^{\circ} \mathrm{C}$ for 30 minutes.

As an early part of our optimization of YBCO films on virgin $\mathrm{MgO}$ substrates, a film growth study was performed to evaluate the effect of sample position relative to the sputtering plasma.

A series of depositions was done where virgin $\mathrm{MgO}<100>$ substrates were placed at various positions within the plasma while maintaining the off-axis relationship of the substrate to the target (Fig. 1). The sample was maintained along a vertical axis at $2.5 \mathrm{~cm}$ from the plane of the sputter target and moved off-axis (upward) at distances ranging from 2.5 to $5 \mathrm{~cm}$. A deposition temperature of $705^{\circ} \mathrm{C}$ was used for this study. We observed a trend of $T_{c}$ increasing from 85 to 92 Kelvin (K) as the substrates were moved away from the RF plasma. Both X-ray $\theta-2 \theta$ scans and STM imagining indicated the presence of a small fraction of aaxis material in the films deposited at the maximum distance while those deposited at the minimum were purely c-axis oriented. There was a correlation between the c-axis lattice parameter measured by X-ray $\theta-2 \theta$ scans and the transition onset temperature showing that films with a c-axis parameter similar to that of bulk

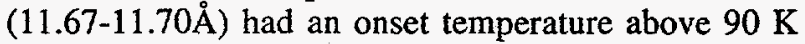
while films with a slightly longer $\mathrm{c}$-axis parameter $(>11.70 \AA$ ) were always less than $90 \mathrm{~K}$. All films used in the studies mentioned hereafter were deposited in that substrate placement that gave us the maximum transition onset in the above deposition series.

The authors [4], and other researchers [5], have previously reported the increase of in-plane 45 degree rotated YBCO grains when using a deposition temperature above $700^{\circ} \mathrm{C}$ on $\mathrm{MgO}<100>$ substrates. In contrast, the crystallinity improved with increased deposition temperature, as shown by X-ray rocking curves and ion beam channeling. To prevent the formation of misoriented grains while achieving high crystallinity, we have found that a film nucleated at temperatures below $695^{\circ} \mathrm{C}$ will establish the desired inplane order, with the subsequent growth completed at higher temperature where superior crystallinity is favored (Fig. 2). The resulting film has no in-plane mis-orientation and a dramatic improvement in the crystallinity as indicated by $\mathrm{X}$-ray rocking curve full width at half maximum (FWHM). X-ray rocking curves on the YBCO (005) reflection of films deposited on virgin substrates, using a low temperature nucleation layer approximately $250 \AA$ thick $\left(20\right.$ minutes @ $670^{\circ} \mathrm{C}$ ) typically showed a FWHM of less than 0.5 degrees when deposited at temperatures from $645^{\circ} \mathrm{C}$ to $725^{\circ} \mathrm{C}$. The series exhibited a temperature dependence with the

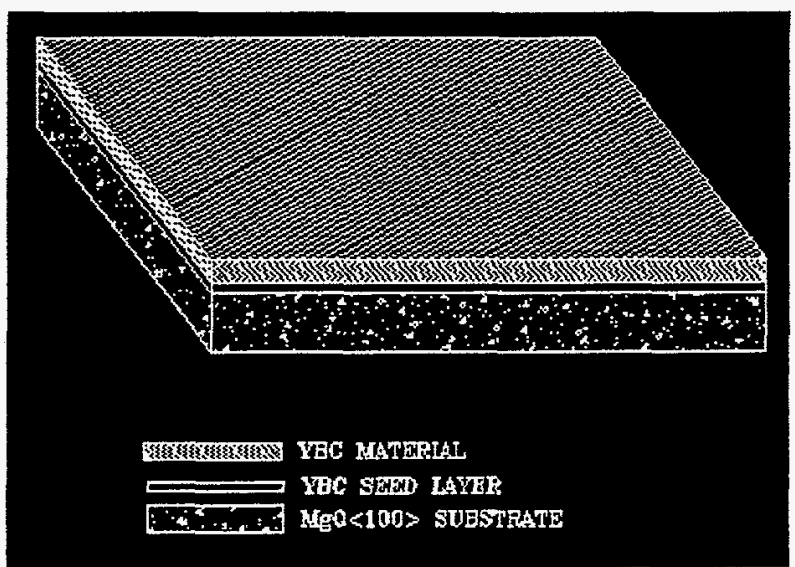


Fig. 2 - YBC film grown using a low temperature nucleated seed layer.

peak widths narrowing to a minimum of 0.2 degrees at the highest temperature. X-ray (018) reflection $\phi$ scan data indicates that there is no 45 degree rotated material in films nucleated at $670^{\circ} \mathrm{C}$ on virgin substrates even when the final deposition temperature was raised to above $720^{\circ} \mathrm{C}$. This procedure could play a major role in the development of YBCO films on $\mathrm{MgO}<100>$ substrates for low surface resistance applications.

Since extremely high quality YBCO can be grown on virgin $\mathrm{MgO}<100>$ substrates by seed layer sputter deposition, a study was performed to see if a similar quality film could be grown on a $\mathrm{MgO}$ substrate after it had been through the processing steps normally required in device fabrication. YBCO films grown on virgin $\mathrm{MgO}<100>$ substrates were argon ion mill etched using an $8 \mathrm{~cm}$ diameter Kaufman-type source to give a processed surface similar to that found in a typical device structure. The optimum ion mill processing parameters for producing high quality $\mathrm{YBCO}$ films were found to be: a beam voltage of $200 \mathrm{~V}$, a beam current of $40 \mathrm{~mA}$ and a beam incidence of 60 degrees from normal. Following the initial removal of the YBCO film, an additional ion mill step for 5 minutes at the same power settings as above, with a normal beam incidence was performed. The samples were then taken immediately from the ion mill chamber to the sputter deposition system. The new YBCO film was then deposited on the processed surface using a low temperature nucleation step as described earlier.

Films deposited on ion milled substrates using a low temperature seed layer had a YBCO (005) reflection FWHM of approximately 1.2 degrees. X-ray $018 \phi$ scan data indicated a small fraction (less than 5\%) of 45 degree rotated grains (Fig. 3). Additionally, neither rocking curve nor $\phi$ scan data indicated a deposition temperature dependence.

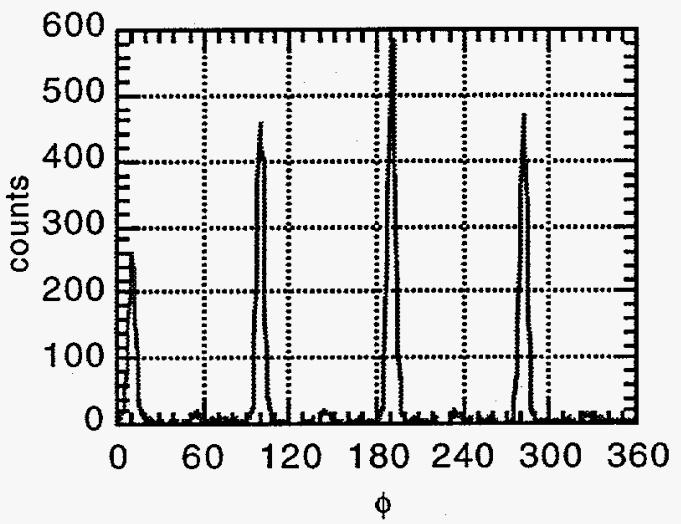

Fig. 3 - X-ray phi scan of a YBCO film deposited using a low temperature seed layer on a processed $\mathrm{MgO}$ substrate.

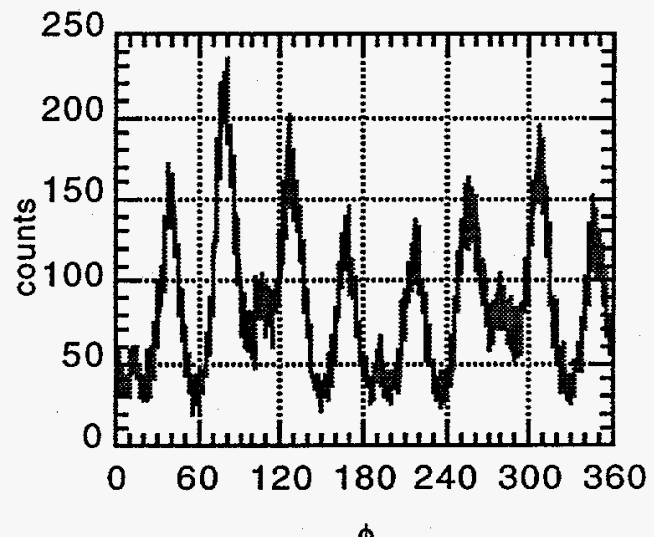

Fig. 4 - X-ray phi scan of a YBCO film deposited without using a low temperature seed layer on a processed $\mathrm{MgO}$ substrate.

When the low temperature nucleation step was altogether eliminated, films deposited on ion milled substrates at deposition temperatures at or above $695^{\circ} \mathrm{C}$ showed a dramatic increase in the YBCO $(005)$ reflection FWHM which was always greater than 2.0 degrees. Also, $x$-ray (018) $\phi$ scan data indicated a high percentage of mis-oriented material (Fig. 4)

DC characterization data showed critical current values in excess of $2.0 \times 10^{6} \mathrm{amps} / \mathrm{cm}^{2}$ at $77 \mathrm{~K}$ for optimum YBCO films deposited on virgin $\mathrm{MgO}$ substrates. The best values we achieved for those on ion milled $\mathrm{MgO}$ were from 0.5 to $1 \times 10^{6} \mathrm{amps} / \mathrm{cm}^{2}$ at $77 \mathrm{~K}$.

For films deposited on virgin $\mathrm{MgO}<100>$ substrates, a minimum RMS surface roughness from $30 \AA$ to $40 \AA$ over a $2 \mu \mathrm{m}$ scan was observed by STM characterization. STM images of YBCO films deposited on ion milled $\mathrm{MgO}<100>$ substrates using a seed layer showed a RMS surface roughness of less than $50 \AA$ over a $2 \mu \mathrm{m}$ scan. A spiral grain microstructure similar to that seen in YBC films deposited on virgin substrates [2] could also be seen on these STM images.

\section{INSULATING LAYERS}

We have established deposition parameters for a variety of insulating oxides including: $\mathrm{CeO}_{2}, \mathrm{LaAlO}_{3}$, $\mathrm{SrTiO}_{3}$, and $\mathrm{MgO}$ to be used in conjunction with YBCO. In our current SNS device program we have chosen $\mathrm{LaAlO}_{3}$ as the material for our insulating layers. In the geometry of our SNS edge junction design, the insulating layer does not have to be epitaxial since the material grown over it was not a functional part of the 
junction. However, we found that a very smooth and uniform insulating layer was advantageous in the processing and materials characterization of the devices. In our best SNS devices we used a $2200 \AA$ thick $\mathrm{LaAlO}_{3}$ insulating layer, deposited insitu over the YBCO layer. The deposition temperature was at $400^{\circ} \mathrm{C}$ in a mixture of 45 milliTorr of Argon with 10 milliTorr of oxygen. Using STM imaging, the resulting film had an RMS roughness of less than $50 \AA$ over a $2 \mu \mathrm{m}$ scan.

\section{NORMAL CONDUCTING MATERIALS}

The identification of normal metallic conducting materials for use with YBCO in the development of high-temperature superconducting (HTS) devices has presented one of the industries more recent challenges. We have established deposition parameters for a variety of these materials including: $\mathrm{La}_{1-x} \mathrm{Sr}_{\mathrm{X}} \mathrm{CoO}_{3}, \mathrm{La}_{1-}$ ${ }_{\mathrm{X}} \mathrm{Sr}_{\mathrm{X}} \mathrm{MnO}_{3}$, and calcium doped $\mathrm{YBCO}$. We have found that all these materials can be grown epitaxially on YBCO and vice versa with no apparent degradation of the HTS properties.

Additionally, in conjunction with our efforts on YBCO film optimization on processed substrates, we have investigated varying the deposition temperature of the YBCO material to produce a normal conductor [6]) at $77 \mathrm{~K}$.

\section{DEVICE CHARACTERIZATION AND DATA}

Several SNS devices have been fabricated using the earlier mentioned deposition methods to produce HTS, Insulator, and $\mathrm{N}$-material films. D.C. characterization of completed SNS junction devices at $77 \mathrm{~K}$ shows a yield of near $100 \%$ RSJ type IV curves from all bridges or SQUIDs on a chip. When microwave power is applied to these junctions, again there is a near perfect yield with most devices showing Shapiro steps. Devices patterned in a SQUID geometry show a SQUID modulation of 10 to $20 \mathrm{mV}$.

\section{CONCLUSION AND DISCUSSION}

The deposition system we have developed is ideally suited for the insitu sputter deposition of HTS materials. We found that a quartz bulb heating a nickel faceplate, and cementing the substrates to the faceplate gave us the best results. While virgin substrates yield the highest quality films, with careful processing of the substrates it is possible to produce high quality $\mathrm{YBCO}$ films on processed $\mathrm{MgO}$ substrates.

YBCO deposited at less than optimum temperature for crystal quality can be used as a nucleation layer for YBCO grown at an optimum temperature. In the final film this accomplishes both improved crystallinity and in-plane orientation. We have found this nucleation step critical to growing high quality films on processed $\mathrm{MgO}<100>$ substrates.

The films deposited in this system are among the smoothest reported to date. The capability to obtain smooth films is essential for the development of HTS devices. The smoothness and homogeneity of these films have have made them ideal specimens in our materials and growth studies.

We thank Daniel Barboa for his efforts in preparing visual aids for the poster presentation that complements this publication. Additionally, we thank Ian Raistrick for his initiation of the microstructure studies of HTS materials on $\mathrm{MgO}$ substrates, leading to these results.

\section{REFERENCES}

[1] B.H. Moeckly, S.E. Russek, D.K. Lathrop, R.A. Buhrman, Jian $\mathrm{Li}$, and J.W. Mayer, "Growth of $\mathrm{YBa}_{2} \mathrm{Cu}_{3} \mathrm{O}_{7}$ thin films on $\mathrm{MgO}$ : The effect of substrate preparation," Appl. Phys. Lett. 57 (16), 15 October 1990.

[2] M. Hawley, I. D. Raistrick, J. G. Beery, R. J. Houlton, "Growth mechanism of sputtered films of $\mathrm{YBa}_{2} \mathrm{Cu}_{3} \mathrm{O}_{7}$ studied by scanning tunneling microscopy", Science Vol. 251, pp. 1587-1589, 29 March 1991.

[3] E.L. Brosha, P.K. Davies, F.G. Garzon, and I.D. Raistrick, "Metastability of superconducting compounds in the $\mathrm{X}-\mathrm{Ba}-\mathrm{Cu}-\mathrm{O}$ system," Science Vol. 260, pp. 196-198, April 9, 1993.

[4] D.W. Reagor, K.N. Springer, F.H. Garzon, M.E. Hawley, R.J. Houlton, C.B. Mombourquette, I.D. Raistrick, and R. Brainard, "Electrical and Structural Properties of $45^{\circ}$ Rotation Grain Boundary Josephson Junctions," MRS Spring Meeting, San Francisco, CA, April 12-16, 1993.

[5] C.B. Eom, J.Z. Sun, B.M. Lairson, S.K. Streiffer, A.F. Marshall, K. Yamamoto, S.M. Anlage, J.C. Bravman, and T.H. Gaballe, "Synthesis and properties of $\mathrm{YBa}_{2} \mathrm{Cu}_{3} \mathrm{O}_{7}$ thin films grown in situ by $90^{\circ}$ off-axis single magnetron sputtering," Physica C 1771, pp 354$382,1990$.

[6] D.W. Reagor, R.J. Houlton, K.N. Springer, M.E. Hawley, Q.X Jia, C.B. Mombourquette, F.H. Garzon, X.D. Wu, "Development of High Temperature Superconducting Josephson Junctions and SQUID's Using Low Temperature $\mathrm{YBa}_{2} \mathrm{Cu}_{3} \mathrm{O}_{7-\mathrm{x}}$ Barriers. Unpublished - Submitted to Applied Physics Letters, September 1994. 